\title{
Performance Benefits of Simultaneous over Sequential Menus as Task Complexity Increases
}

\author{
H. Hochheiser, N. Kositsyna, G. Ville*, B. Shneiderman* \\ Human-Computer Interaction Lab, Dept. of Computer Science, \\ * Institute for Systems Research \& \\ Institute for Advanced Computer Studies \\ University of Maryland, College Park, MD 20742 USA
}

\begin{abstract}
To date, experimental comparisons of menu layouts have concentrated on variants of hierarchical structures of sequentially presented menus. Simultaneous menus - layouts which present multiple active menus on a screen at the same time - are an alternative arrangement that may be useful in many web design situations. This paper describes an experiment involving a between-subject comparison of simultaneous menu and their traditional sequential counterparts. Twenty experienced web users used either simultaneous or sequential menus in a standard web browser to answer questions based on US Census data. For novice users performing simple tasks the simplicity of sequential menus appears to be helpful, but for most tasks and most users there is good evidence to believe that simultaneous menus speed performance and improve satisfaction. Design improvements can amplify the benefits of simultaneous menu layouts.
\end{abstract}

\section{Keywords}

Menu Design, Sequential Menus, Simultaneous Menus, Experimental Comparison

\section{Introduction}

Over the past fifteen years, researchers have revisited the subject of menu design from a variety of perspectives. Numerous experimental studies have been conducted in an ongoing attempt to clarify tradeoffs between depth, breadth, and cognitive load. Studies such as [4] and [3] established two competing views: while Landauer and Nachbar used a model based on Fitt's Law to argue for increased breadth over depth, Kiger argued that menu breadth should be limited to eight or nine selections at each level. Subsequent studies have shown strong benefits for broad menu trees with fewer levels [6], most recently in the context of web page design [5]. The debate rages on, but evidence increases that shows a strong relationship between menu depth and task performance times $[10,14]$, and many successful commercial websites begin with home pages that reduce menu depth by having over one hundred links. In any case, it is clear that the processes involved are not completely understood: recent modeling work suggests that cognitive aspects of menu search may be more complicated than originally thought [2]. 
Much of this research involves the use of hierarchical or sequential layouts (Figure 1). These menus present choices that must be made in some predetermined order, with the impact of a given choice constrained by the sum total of all previous choices. This strategy is often appropriate, particulary for situations requiring context-dependent menu choices, such as choosing a continent, then a country, then a city, to get a list of tourist attractions. However, the rigidity of hierarchical menus causes difficulties for some tasks, particularly when comparisons among the results of multiple selections are required. To complete such tasks with a hierarchical menu layout, users must make repeated choices involving repeated backtracking through the hierarchy.

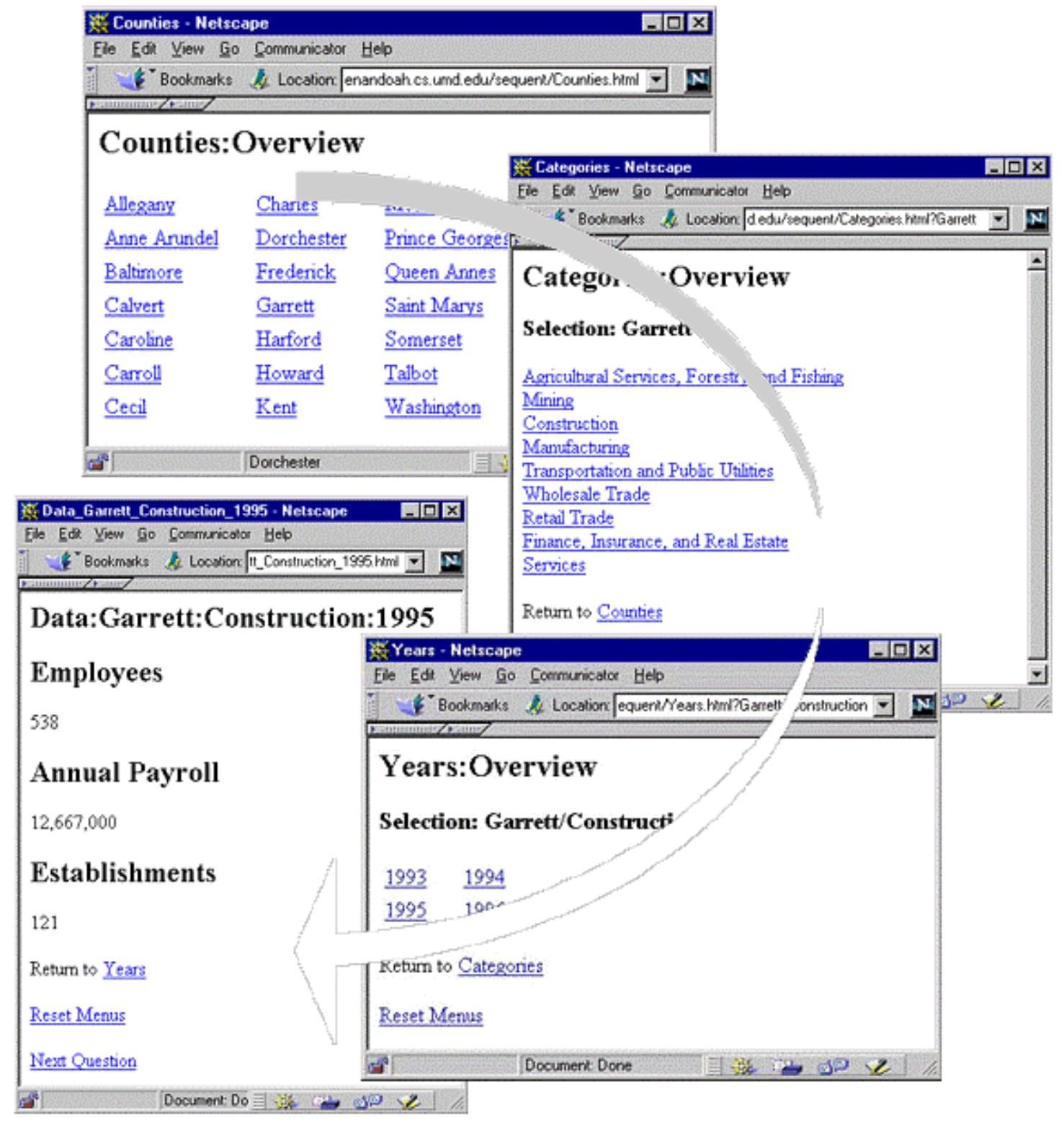

Figure 1: Sequential Menus. Users must make once choice from each menu in succession. Users can select the "Return to ..." links to revisit the previously displayed menu, or the "Reset Menus" link to return to the first menu selection. 
Simultaneous menus (Figure 2) are an alternative design possibility appropriate for tasks that do not involve context-dependent modification of menu contents. These menus, which simultaneously display choices from multiple levels in the hierarchy, provide users with the ability to make choices from the menu in any order, for example choosing continents, primary language, and types of tourist attractions to get a list of cities with their attractions. This flexibility may lead to improved performance or user satisfaction for some tasks.

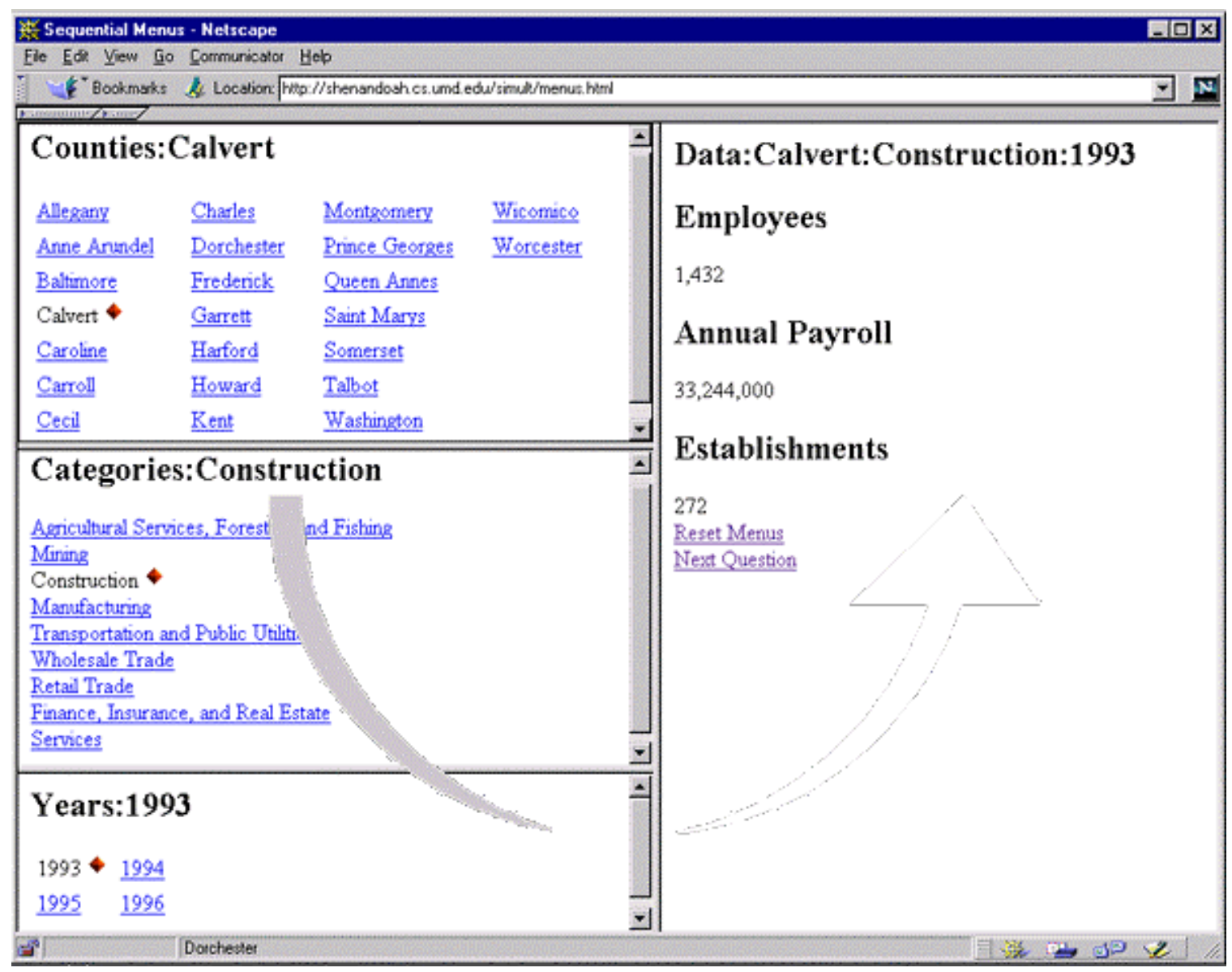

Figure 2: Simultaneous menus. Users can choose from any one of the three menus on the left at any point in time.

While systems such as the National Digital Library collection browser [9] and Spotfire [13] used simultaneous menus, evaluation has been limited. One study found that tasks involving simultaneous menus took less time and had fewer errors than tasks involving hierarchical menus [12]. The authors of this study hypothesized that the use of a stable spatial presentation of the menus eliminated the need for repeated visual scanning, thus offsetting any increased cognitive load caused by a larger display.

Simultaneous menus are not without drawbacks. Effective use of this strategy depends upon the availability of screen real estate necessary for display of the appropriate menu 
choices, so simultaneous menus may not be appropriate for very broad (or very deep) menu structures. Furthermore, simultaneous menu structures that display large amounts of information over a significant portion of available screen space may require additional mouse movement and/or cognitive processing that could offset improvements in performance. The choice between these two presentation styles is complex and taskdependent.

However, smultaneous menus are especially advantageous in a web environment since they reduce the number of time consuming web accesses compared with sequential menus. We wished to avoid these variable delays in our study and to demonstrate that simultaneous menus had advantages for general purpose use.

After an initial discussion of a model for performance times using the two menu layouts, this paper presents an experiment that compares user task performance times for sequential vs. simultaneous menus. Our hypotheses were that simultaneous menus will have faster performance times and greater user satisfaction than sequential menus. Furthermore, the performance advantage of simultaneous menus should increase with the number of menu choices required to complete a task.

\section{A Task-Based Predictive Model}

Intuitively, the simultaneous menu layout would appear to have the advantage of freeing users from making selections in a pre-determined order. For simple tasks involving a single selection from each menu, this may lead to a significant performance improvement. However, the real benefits of simultaneous menus are likely to be seen in tasks that require revisitation of menus in order to compare results of different choices.

To see why this is so, we imagine a set of three menus, and three types of questions, involving varying levels of difficulty. The simplest task requires one selection from each of the three menus. The second task requires comparison between two selections, which differ only in the choice made from the third menu. To complete this task, the user must traverse the menu tree once, note the appropriate result, and make a second selection from the last menu to select the appropriate comparison data. The third task is similar to the second, but the selections differ only in the choice made from the second menu.

For simultaneous menus, these more complicated tasks involve minimal additional overhead: as all menus are constantly available, the user can simply move to the appropriate menu and make the desired choice. However, these tasks place sequential menus at a significant disadvantage, as users must explicitly "backtrack" to return to a previously displayed menu and make a new choice. Thus, our expectation was that simultaneous menus would lead to faster task performance than sequential menus and that performance advantages of simultaneous menus would be greater for tasks involving more backtracking through the menu structure.

A simple "clicks model" [1], based on the number of clicks required for each task type, will provide a more specific understanding of the predicted performance differences: 
- Type 1: For both menu layouts, users must make one selection in each of the three menus, for a total of three clicks.

- Type 2: Users must make one selection at each of the three levels, plus appropriate clicks to get the second data point. For simultaneous menus, this involves one additional click on the third menu, for a total of 4 clicks. For sequential menus, one click of the "Back" button is required, along with one additional click on the third menu, for a total of 5 .

- Type 3: For simultaneous menus, four selections are necessary, as these questions only alter one of the three menu choices. However, sequential menus require 7 clicks: 5 as required for type two, plus one Back click and a new menu selection at the second level.

These results are summarized in table 1. The "Items Changed" for any given task is the total number of menu choices that changes. For type three, two backtracking steps are required in the sequential case even though only one item is varied: these questions require a different choice on the second menu, while requiring the same choice for both visits to the third menu. Thus, these questions are somewhat easier for simultaneous menu users, who need only make one additional selection from the second menu to complete the task.

\begin{tabular}{l|llll} 
Task & \# Items Varied & $\begin{array}{l}\text { \# Backtrack } \\
\text { Steps }\end{array}$ & $\begin{array}{l}\text { Simultaneous } \\
\text { Clicks }\end{array}$ & $\begin{array}{l}\text { Sequential } \\
\text { Clicks }\end{array}$ \\
\hline Type 1 & 0 & 0 & 3 & 3 \\
Type 2 & 1 & 1 & 4 & $5(=3+1+1)$ \\
Type 3 & 2 & 2 & 4 & $7(=3+2+2)$
\end{tabular}

Table 1: Summary of the three task types. Type 1 included no backtracking, so three clicks were needed for both sequential and simultaneous menus. Type 2 questions involved two choices from the third menu, thus requiring two additional clicks for sequential menu users (one to return to the previous menu, and one to make a second choice), and one additional click for simultaneous menus. Finally, type 3 questions varied the second category. For simultaneous menus, this added only the one click required to make the additional choice. However, sequential users had to make 4 additional clicks: two to return to the second menu, one to make a new choice from that menu, and one to repeat the selection made from the third menu.

Further analysis can generalize the contents of Table 1 into a predictive model based on the number of clicks required to complete each task. For simultaneous menus, users must make one selection at each of the initial menus, followed by an additional click for each comparison that must be made. If we refer to the result of one complete traversal of the menu sequence as a single data point, the total number of comparisons that must be made is one less than the number of data points that must be accessed: to make one comparison, I must access two data points, etc . Thus, tasks involving the use of $d$ simultaneous menus involving to compare data from $s$ data points will require a total of $d+s-1$ clicks.

For sequential menus, the number of backtracking steps is the crucial factor in determining the number of clicks rqeuired to complete a task. To see why this is so, we 
first note that one choice from each menu will be necessary to view the first data screen. After those choices are made, each backtracking step involves two additional clicks: one to return to the previous menu, and a second click to make a selection from the menu to which the user was backtracking. Thus, $b$ backtracking steps require $2 b$ additional clicks, for a total of $d+2 b$ clicks ( $d$ is the number of menus, as above.).

For both menu types, we assume that each menu selection action takes a given time - $t_{\text {sim }}$ for simultaneous menus or $t_{\text {seq }}$ for sequential. Each task involves a constant (possibly zero) initiation time - $k_{\text {sim }}$ or $k_{\text {seq }}$. Combining these observations, we derive the following equations:

Simultaneous: $\quad T_{\text {sim }}=(d+s-1) t_{\text {sim }} c_{\text {sim }}+k_{\text {sim }}$,

Sequential: $\quad T_{\text {seq }}=(d+2 b) t_{\text {seq }} c_{\text {seq }}+k_{\text {seq }}$

As above, $d$ is the number of menus (three in our examples), $s$ is the number of screens that must be compared in the simultaneous case, $b$ is the number of backtracking steps required in the sequential case, and $c_{\text {sim }}$ and $c_{\text {seq }}$ are constants determined by the type of menu layout being used (sequential or simultaneous). Expanding terms, these equations become:

Simultaneous: $\quad T_{\text {sim }}=s t_{\text {sim }} c_{\text {sim }}+(d-1) t_{\text {sim }} c_{\text {sim }}+k_{\text {sim }}$

Sequential: $\quad T_{\text {seq }}=2 b t_{\text {seq }} c_{\text {seq }}+d t_{\text {seq }} c_{\text {seq }}+k_{\text {seq }}$

For any given set of menus, the second and third terms of these equations will be constants.

These models assume that all clicks take approximately equal amounts of times. A more complete model would include predictors for times required to make choices from each of the $d$ menus. Such a model would built upon research showing that menu selection times can be roughly logarithmic or linear $[4,7,11]$. Although we expect that selection times for individual menus used in this experiment will conform to these earlier findings, item selection times for individual menus may differ when used in different layouts. Specifically, the increased amount of information on the simultaneous menu screen may lead to greater cognitive load, causing item completion times to be greater than for sequential menus. However, users of sequential menus may be slowed by the increased number of re-orientations required.

\section{Experiment}

Informal investigation and the above predictive model led us to hypothesize that users of simultaneous menus would be able to complete tasks in less time than users of comparable sequential menu layouts. Furthermore, simultaneous menus should show increasing performance advantages as task complexity increases. Although other 
dependent factors - specifically learning time and accuracy - might be measured, they were not addressed in the current experiment.

Our experiment used the three question types described above to provide three separate types of tasks. Each participant answered questions using one of the two menu layouts. The experimental task consisted of 15 questions, divided evenly among the three types described above. Task completion times were aggregated by menu type and task type, and mean types for the three types were compared by menu layout. Thus, each task type was considered a separate, between-subject, experimental group, allowing the use of three separate, one-tailed $\mathrm{T}$-tests for statistical validation at the 0.05 level of significance.

Experimental tasks were based on data taken from the U.S. Census Bureau's MapStats web page (http://www.census.gov/datamap/www/index.html). County business patterns profile data for 1993-1996 provided a data set covering 23 counties, nine industries, and four years. These attributes formed the basis for a three-menu layout, with the sequential menu layout displaying counties first, industries second, and years third.

Each combination of county, industry, and year had three corresponding facts: annual payroll, number of employees, and number of establishments. This formed the basis for the questions, which required retrieving individual facts ("How many people were employed in Kent County in service businesses during 1993?") or comparing between two different data points ("Which business category employed the larger number of people in Howard County in 1995: manufacturing or wholesale trade?”).

The experiment involved a total of 21 questions, split evenly among the three question types described in section 2. Thus, one-third of the questions required one selection from each of the three menus, one-third required an additional selection from the third (year) menu, and the remainder required an additional selection from the second (industries) menu. Of the 21 questions, six were practice questions and experimental data was taken from the 15 remaining questions. Practice and experimental questions were presented in a balanced order consisting of sets of three questions, with each set containing one question of each type.

The menus were presented to users as HTML hyperlinks displayed in a Netscape browser. In the simultaneous menu case (Figure 2), menus were displayed in three frames on the left-hand side of the browser window, while a frame on the right-hand side contained the results, or text asking the user to make a choice from any menus that have not yet been selected. After selections were made, the relevant menu windows would refresh to highlight the selected item. At any time, the user had the option of selecting the "Return to Start" link, which would reset the menus to their original configuration.

Sequential menus (Figure 1) were presented in a series of three screens, each containing one of the three menu items. Users moved forward in the menu sequence by simply selecting a single item from a menu. Two types of links supported returning to previously viewed menus in the sequence: a "Return to Start" link cleared the selection state of the system and returned to the initial menu screen, while a "Return to ..." link on the second 
and third menu screens was available for moving back to the previously displayed menu. All menu screens (with the exception of the first menu) contained feedback mechanisms summarizing the choices that had been made on previous menus.

In order to eliminate variation due to network delays, files were served from an Apache web server running locally on the machines used for testing. Browser cache functionality was disabled, in order to guarantee that each menu request generated a page request to the server. As this configuration gurantees an entry in the server log file for each menu selection, request timestamps in the server logs were used to extract task performance times.

Twenty-two subjects participated in the experiment, but data for two subjects were not used due to task completion times that were several times greater than the others. Of the remaining twenty subjects, 15 were male, 5 were female, and all were under 45 years of age. All subjects were graduate or undergraduate students, and all had previous webbrowsing experience. Subjects were randomly assigned to use either sequential or simultaneous menus: of the twenty data sets used in the analysis, eleven involved simultaneous menus while the other nine used sequential menus.

Participants began their experimental sessions by signing the consent form, completing the background questionnaire, and reading a one-page instruction document appropriate for the menu layout being used. After indicating their understanding of the instructions, users completed the practice tasks, took a short break if needed, and proceeded to the experimental tasks. Finally, users completed a short post-experimental questionnaire aimed at understanding their subjective reactions.

Task presentation and completion were handled identically for both menu layouts and phases of the experimental session (practice and experimental questions). All questions were presented to the user on a sheet of paper, which was also used to record the answers. Each task began with the browser screen on a page containing a single link labelled "Next Question". Users were instructed to read the question first, and to select the link only after they had completely read the question. Selection of this link led to display of the appropriate menu screen, allowing the users to navigate the menus to find the appropriate data. Users were instructed to continue until they found the information needed to answer the question, at which point they should write the answer on the sheet. After writing the answer, users chose the link marked "Next Question", which returned the browser to the initial start page, ready for the next question. The elapsed time between the selection of the "Next Question" links was recorded as the time required to complete the task. The instructions, task presentation, and menu layout were all revised to account for feedback from a pilot test with four subjects.

This experiment measures task performance times for users who are unfamiliar with simultaneous menus. In order to understand the potential performance as users become more comfortable with simultaneous menus, three of the authors completed the experimental tasks three times for each menu layout. The best time for each question was extracted from the resulting data set, creating a projected performance profile. Although 
less formal and thorough than an experimental evaluation of learning effects, this analysis provides some insights into the possible benefits of simultaneous menus for experienced users.

\section{Results}

Table 2 and Figures 3 and 4 summarize the results.

\begin{tabular}{l|lllllll}
$\begin{array}{l}\text { Question } \\
\text { Type }\end{array}$ & Menu Layout & Min & Max & Average & $\begin{array}{l}\text { Std. } \\
\text { Dev. }\end{array}$ & $\begin{array}{l}\text { "Experienced } \\
\text { User" } \\
\text { Average }\end{array}$ & $\begin{array}{l}\text { "Experienced } \\
\text { User" } \\
\text { Std. Dev. }\end{array}$ \\
\hline Type 1 & Sequential & 5 & 36 & 14.6 & 5.9 & 8.4 & 1.3 \\
& Simultaneous & 12 & 43 & 21.3 & 6.1 & 7.8 & 1.5 \\
Type 2 & Sequential & 9 & 41 & 25.1 & 8.1 & 15.0 & 1.4 \\
& Simultaneous & 12 & 72 & 29.3 & 8.7 & 10.2 & 1.6 \\
Type 3 & Sequential & 12 & 69 & 39.4 & 12.7 & 22.2 & 1.3 \\
& Simultaneous & 15 & 62 & 33.5 & 9.6 & 13.2 & 1.8
\end{tabular}

Table 2: Summary of Task Completion Times. For the experimental results, sequential menus were faster for types one and two, and simultaneous menus were faster for type three. For the "experiencd user" results, simultaneous menus were always faster. In all cases, task completion times increased as complexity increased.

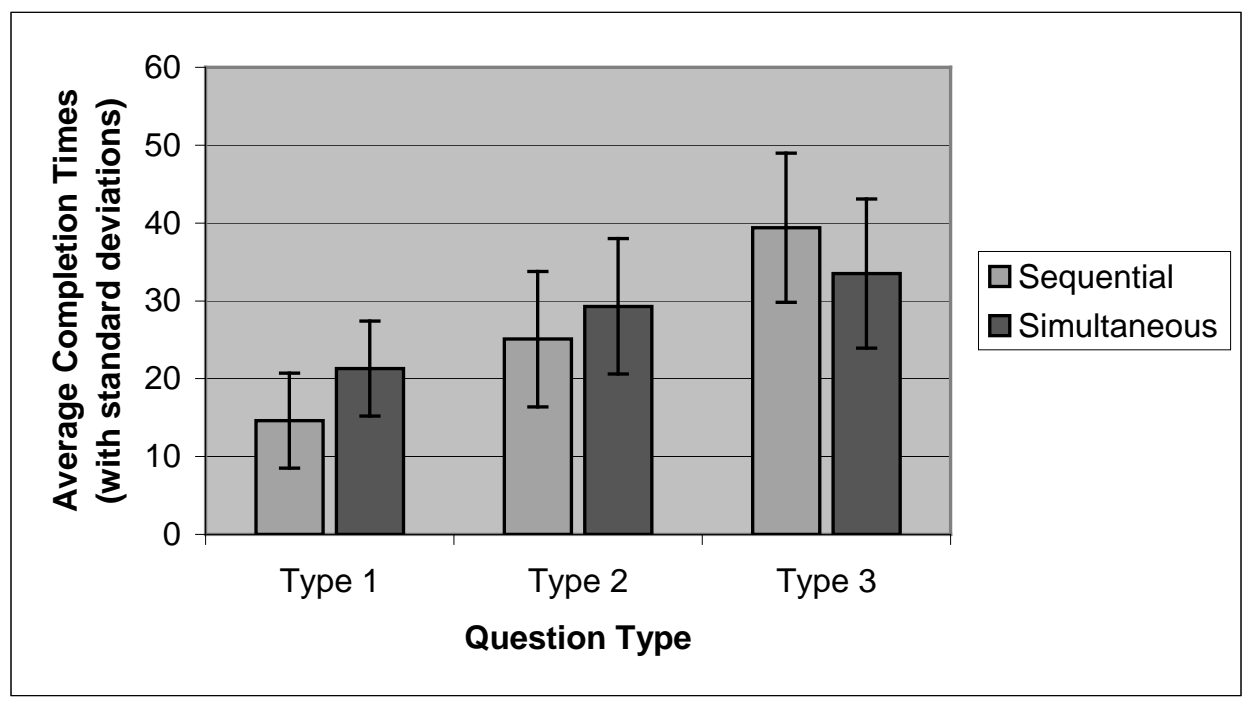

Figure 3: Experimental Results: For the experimental tasks, sequential menus provided better performance for types one and two, but simultaneous menus were faster for type three. Error bars indicate a range of one standard deviation from the mean. 


\begin{tabular}{l|lllll} 
Task Type & $\begin{array}{l}\text { Sequential Menu } \\
\text { Observations }\end{array}$ & $\begin{array}{l}\text { Simultaneous Menu } \\
\text { Observations }\end{array}$ & Df & t-stat & P(T t) one-tail \\
\hline Type 1 & 45 & 55 & 98 & -5.49 & $<0.001$ \\
Type 2 & 45 & 55 & 98 & -2.45 & 0.008 \\
Type 3 & 45 & 55 & 98 & 2.65 & 0.005
\end{tabular}

Table 3: T-Test Results: For types one and two, sequential menus are significantly faster. However, for type three, the simultaneous menus are significantly faster.

Results of statistical analyses of the experimental data are given in table 3. For question types 1 and 2, the sequential menus were significantly faster. However, simultaneous menus were significantly faster for type three questions ( $\mathrm{P}<0.05$ in all three cases). This change appears to indicate that the performance advantages of simultaneous menus increase with the number of backtracking steps required. This data includes times for incorrect responses: we assume that the subjects took the time and did the appropriate page navigation even if the final answer was incorrect.

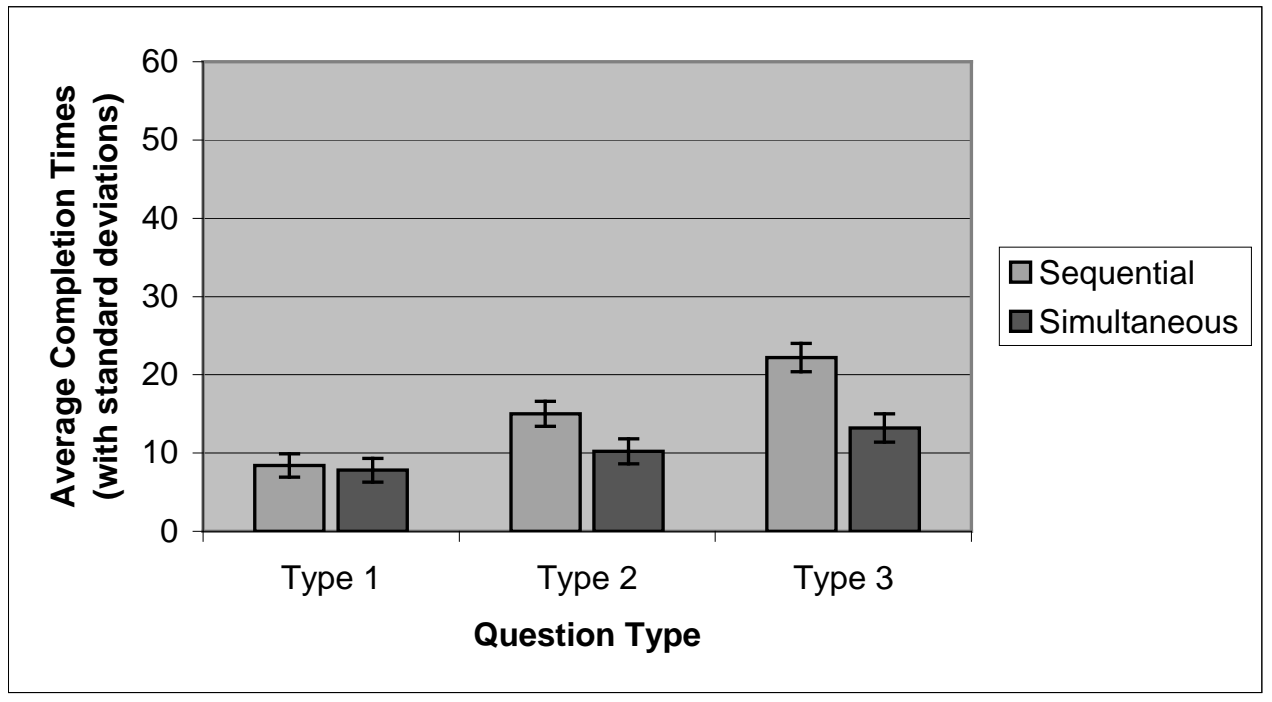

Figure 4: Projected performance results for experienced users: Simultaneous menus are faster for all three types of questions, indicating a possible learning effect that may favor simultaneous menus.

Results from the projected performance of experienced users are given in figure 4 . Simultaneous menus outperformed sequential menus for all three task types, and performance differences increased with task complexity.

The results of the post-experiment questionnaire are compiled in table 5 . 


\begin{tabular}{llll}
\hline Questions & Scale (1-9) & Sequential ( $\mathrm{n}=9$ ) & Simultaneous ( $\mathrm{n}=11$ ) \\
\hline System & Frustrating-satisfying & 5.89 & 6.36 \\
& Difficult-easy & 6.78 & 7.27 \\
Helpful layout & Never-always & 5.78 & 6.45 \\
Information arrangement Illogical-logical & 6.89 & 6.90 \\
& Confusing-easy & 8.56 & 7.27 \\
Amount of information & Inadequate-adequate & 5.56 & 7.00 \\
Navigation & Difficult-easy & 6.44 & 7.91 \\
Initial instruction & Inadequate-adequate & 8.22 & 7.55 \\
\hline
\end{tabular}

Table 5: Subjective Questionnaire Results: Roughly comparable subjective responses to the two menu types provide some indication that users are not necessarily confused or disoriented by simultaneous menus.

Subjective responses to the two menu types were similar. Users found both simultaneous and sequential menu layouts to be somewhat satisfying, easy to use, and easy to navigate. Although not statistically meaningful, the simultaneous menus were rated somewhat higher in terms of "helpful layout", and somewhat lower in terms of "information arrangement". Additional evaluation - particularly involving individuals with less computer experience - may clarify user preferences, but the lack of a clear trend of confusion or disorientation among users of the simultaneous menu layout is encouraging.

\section{Predictive Model Revisited}

Our models assume a single item selection time for all menus used in a given layout: for any single layout (simultaneous or sequential), selection times for the three menus (counties, industries, and years) should be comparable. For both sequential and simultaneous windows, analysis of data for the individual menus showed similar item selection times. In both cases, selection times for the county and year menus were significantly shorter than times for the industry menus, and no significant differences between the county and year menus were observed. This result is somewhat surprising, since the industry menu had fewer items (9) than the county menu (23). The cognitive load involved in processing the menu choices may have been a factor: while county names are short (one or two words) and possibly familiar, industry names involved greater amounts of text with which participants were less likely to be familiar. In the absence of any evidence of a relationship between menu lengths and item selection times, the assumption of a single item selection time for each layout seems justifiable.

To examine the fit between our data and the predictive models given above, we conducted a regression of the task completion times against the number of data points compared (for simultaneous menus), or the number of backtracking steps required (for sequential menus). For the simultaneous menus, the linear regression equation was $T_{\text {sim }}=11.4+10.0 \mathrm{~s}, r^{2}=0.24$. For sequential menus, the linear equation was $T_{\text {seq }}=14.0+12.4 b, r^{2}=0.54$. The data plot given in Figure 3 hints at the possibility of a 
non-linear effect for sequential menus. The corresponding quadratic regression equation is $T_{\text {seq }}=14.6+8.6 b+1.9 b^{2}, r^{2}=0.54$.

On average, the choice time required for a menu in a simultaneous layout is greater than the time required for the same menu in a sequential layout. We can use the average menu selection times for each of the menu layouts to relate the linear equations back to the predictive models presented above. For simultaneous menus, the average selection time was 4.9 seconds $\left(t_{\text {sim }}=4.9\right)$, and the average for sequential menus was 3.0 seconds $\left(t_{\text {seq }}=3.0\right)$.

Using these values, and the depth of the menu structure used ( $d=3$ ), we can identify the constants that match the calculated regressions. Specifically, for the simultaneous menus, we find $c_{\text {sim }}=2.0$ and $k_{\text {sim }}=-8.2$, so $T_{\text {sim }}=2.0 t_{\text {sim }} s+(2 * 2.0) t_{\text {sim }}-8.2$. Using the average value $t_{\text {sim }}=4.9$, this becomes $T_{\text {sim }}=2.0 t_{\text {sim }} s+11.4$, where $s$ is the number of data points visited. Similarly, for sequential menus, we find that $c_{\text {seq }}=2.1$ and $k_{\text {seq }}=-4.6$, so $T_{\text {seq }}=(2 * 2.1) b t_{\text {seq }}+(3 * 2.1) t_{\text {seq }}-4.6$, or $T_{\text {seq }}=4.1 t_{\text {seq }} b+14.0$, where $b$ is the number of backtracking steps required to complete a task.

Although these results support the use of a clicks-only predictive model, further work will be needed to validate these models. Specifically, the non-linear effect in the sequntial menu data suggests that the advantage for simultaneous menus grows as tasks become more difficult. We believe that this is the result of the increased cognitive load of repeated backtracking while comparing multiple data points, which is likely to be difficult for sequential menu users.

Additional experiments involving a wider range of backtracking steps and required mouse clicks might clarify the time functions for both menu types. More accurate accounting for the time to read menus and make choices could lead to a deeper understanding of the components of task completion times. Inclusion of appropriate models of mouse motion and distance (perhaps based on Fitts' Law) could account for the effects of screen layout. Finally, investigations of learning rates could lead to models that predict improvements in task performance.

\section{Discussion}

Sequential menus provided significantly superior performance only for questions that did not require multiple selections from any given menu (Type 1). For tasks that involved progressively greater amounts of backtracking, performance with simultaneous menus improved relative to that of sequential menus, leading to a statistically significant difference for the most complicated questions (Type 3). Our experienced user performance profile provided further evidence supporting the use of simultaneous menus. These advantages are likely to increase in web environments where network access times will still further delay users of sequential menus. 
While these results provide evidence that simultaneous menus are appropriate in certain contexts, we were surprised by the superior performance of sequential menus for the Type 1 tasks involving zero or one backtracking steps. Artifacts of our experimental design may have influenced these results:

Screen Layout: For the sequential menus, each menu appeared in the screen in the space occupied by the previous menu. This layout minimized the mouse movement required: all tasks could be completed in the upper left quadrant of the screen. On the other hand, the simultaneous menus were displayed in a vertical column of three frames, occupying the entire left half of the screen. The extra movement required may have degraded performance. Compact menu arrangements could reduce the extra mouse movement required for navigation of simultaneous menus.

Familiarity of Menu Presentation Style: Since most of the participants were undergraduate and graduate students with substantial experience using web browsers, it seems reasonable to conclude that the sequential menu format was well understood. As simultaneous menus are unfamiliar, there may be a learning effect involved in the results.

Task Choice: All three task types in this experiment involved closed-end questions with known answers. However, the performance advantage of simultaneous menus relative to sequential menus increased with the amount of backtracking required. Repetition of this experiment with tasks that involve more backtracking may lead to results that are still more favorable towards simultaneous menus.

Simultaneous menus fared well on the post-test subjective questionnaire: ratings for the two menu arrangements were roughly comparable on all of the subjective questions. Since each subject used only one of the two menu presentation styles, a true preference comparison between the two styles is not possible. Further study involving withinsubjects comparison of the two menu styles might clarify issues related to user preference while providing additional data for performance comparisons.

\section{Design Implications}

When sequential menu hierarchies can be converted to simultaneous menu presentation, this strategy should be considered, especially if exploratory tasks are anticipated. Simultaneous menus show users all alternatives at all levels at once, thereby aiding comprehension of all possibilities, although the increased perceptual and cognitive load may slow novice users in simple tasks.

Simultaneous menus usually require more display space, which may render them inappropriate for certain display environments and menu structures. This increased screen content may lead to further increases in perceptual load, which could have additional negative effects. Compact presentation formats might be used to present simultaneous menus in a manner that minimizes these detrimental effects. Interface widgets such as sliders or checkboxes may provide more effective and compact representations of menu choices. 
The user population may influence the choice between simultaneous and sequential menus. Our experimental data, which was collected from subjects unfamiliar with simultaneous menus, showed an advantage for sequential menus on simpler tasks. However, this advantage disappeared in our projected profile based on more experienced users, suggesting that the benefits of simultaneous menus are likely to increase with user experience.

\section{Conclusion}

We have shown that simultaneous menus can lead to improvements in user performance over comparable sequential layouts. The choice between simultaneous and sequential menu layouts should be made on the basis of the expected task: if users are expected to make multiple selections from two or more menus, simultaneous menus provide better performance. This advantage will be amplified in web environments that incur delays for each network access. Simultaneous menus appear to be well-suited for exploratory tasks, since they also provide a continuous overview of menus at all levels.

Much of the menu design literature has focused on analysis of the breadth vs. depth question in hierarchical menu structures. Although clearly important, these investigations present an overly simplistic view of the problem of menu structure design. Our study presents one alternative to strictly hierarchical menus, along with evidence that simultaneous menus can lead to improved performance.

Comparisons that limit the parameters of menu designs to depth and breadth may not account for some factors that affect performance. Studies of menu structures with different shapes [8] and with differing amounts of contextual information [1, 15] have shown that performance can be influenced by the type of task, the amount of context given, and the shape of the menu. Examination of these issues as they apply to simultaneous menus may be a promising direction for future work.

\section{Acknowledgements}

Thanks to Kent Norman for his assistance with experimental design and statistics. Richard Salter provided invaluable assistance with HtX (http://www.cs.oberlin.edu/ rms), which proved indispensable for generation of the HTML pages needed to run the experiment. Finally, we appreciate the partial support of the US Census Bureau and IBM's University Partnership Program.

\section{References}

[1] Chimera, R. and Shneiderman, B. An exploratory evaluation of three interfaces for browsing large hierarchical tables of contents. ACM Transactions on Information Systems 12, 4 (October 1994), 383-406.

[2] Hornof, A. and Kieras, D. Cognitive modeling reveals menu search is both random and systematic. In Proc. CHI '97 Conference: Human Factors in Computer Systems, ACM Press, NY (1997), 107-114. URL http://www.acm.org/sigchi/chi97/proceedings/paper/ajh.htm. 
[3] Kiger, J. The depth/breadth trade-off in the design of menu-driven user interfaces. International Journal of Man-Machine Studies 20 (1984), 201-213.

[4] Landauer, T. and Nachbar, D. Selection from alphabetic and numeric trees using a touch screen: Breadth, depth, and width. In Proc. CHI '85 Conference: Human Fators in Computer Systems, ACM Press, NY (1985), 73-78.

[5] Larson, K. and Czerwinski, M. Web page design: Implications of memory, structure, and scent for information retrieval. In Proc. CHI '98 Conference: Human Factors in Computer Systems, ACM Press, NY (1998), 25-31.

[6] Lee, E. and MacGregor, J. Minimizing user search time in menu retrieval systems. Human Factors 27 (1985), 157-162.

[7] Norman, K. The Psychology of Menu Selection: Designing Cognitive Control of the Human/Computer Interface. Ablex Publishing Corporation, Norwood, NJ (1991).

[8] Norman, K. and Chin, J. The effect of tree structure on search in a hierarchical menu selection system. Behaviour and Information Technology 7, 1 (1988), 51-65.

[9] Plaisant, C., Marchionini, G., Bruns, T., Komlodi, A., and Campbell, L. Bringing treasures to the surface: Iterative design for the library of congress national digital library program. In Proc. CHI '97 Conference: Human Factors in Computer Systems, ACM Press (1997), 518-525.

[10] Salvendy, G. and Jacko, J. Hierarchical menu design: Breadth, depth, and task complexity. Perceptual and Motor Skills 82 (1996), 1187-1201.

[11] Sears, A. and Shneiderman, B. Split menus: Effectively using selection frequency to organize menus. ACM Transactions on Human-Computer Interaction 1, 1 (1994), 2751.

[12] Seppala, P. and Salvendy, G. Impact of depth of menu hierarchy on performance effectiveness in a supervisory task: Computerized flexible manufacturing system. Human Factors 27 (1985), 713-722.

[13] Spotfire. Spotfire [WWW Document] (1999). URL http://www.spotfire.com.

[14] Zaphiris, P. and Mtei, L. Depth. vs. breadth in the arrangement of web links (1997). URL http://otal.umd.edu/SHORE/bs04.

[15] Zaphiris, P., Shneiderman, B., and Norman, K. Expandable indexes versus sequential menus for searching hierarchies on the world wide web, (1999). URL http://www.kypros.org/ pzaphiri/Paper. 